\title{
New method for determining the degree of fibrillation of regenerated cellulose fibres
}

\author{
Yibo Ma (1) - Marja Rissanen • Xiang You • Kaniz Moriam • Michael Hummel • \\ Herbert Sixta
}

Received: 18 August 2020/Accepted: 4 October 2020/Published online: 24 October 2020

(C) The Author(s) 2020

\begin{abstract}
In this study, we propose a convenient method for testing the fibrillation tendency of manmade cellulosic fibres (MMCFs) and investigate the possibility to apply a commercial crosslinker for Tencel fibres on the ionic liquid-based regenerated cellulosic fibre (Ioncell fibre). The fibrillation tendency of various MMCFs including viscose, Modal, Tencel and Ioncell fibres were examined through wet abrasion by using ball bearing and blending methods. The fibrillation tests using a laboratory blender was found to be a superior method over the ball bearing method in terms of time and energy saving. The fibrillation tendency of the fibres highly depended on their cellulose molecular orientation and the treatment intensity (time, temperature and alkalinity) in the blender. This fibrillation method was also applied to discover the effect of the crosslinking on the fibrillation tendency of the fibres. The Ioncell fibre proved to be suitable for crosslinking treatment to reduce fibrillation using 1,3,5-triacryloyl-hexahydro-1,3,5triazine (TAHT) — a commercial Tencel crosslinker.
\end{abstract}

Y. Ma $(\bowtie) \cdot$ M. Rissanen $\cdot$ X. You ·

K. Moriam · M. Hummel · H. Sixta $(\bowtie)$

Department of Bioproducts and Biotechnology, School of Chemical Engineering, Aalto University, P.O. Box 16300, 00076 Aalto, Finland

e-mail: yibo.ma@aalto.fi

H. Sixta

e-mail: herbert.sixta@aalto.fi
Keywords Regenerated cellulose fibre - Tencel · Ioncell $\cdot$ Crosslinking $\cdot$ Fibrillation

\section{Introduction}

Man-made cellulosic fibres (MMCFs) is a class of fibres that are converted from cellulosic dissolving pulps via dissolution and fibre spinning technologies. They have certain physical properties that are superior compared to the synthetic fibres, such as excellent moisture management and breathability. MMCFs are considered to be important add-ons to the cellulose textile market due to the growing demanding of the commercial textile products and the stagnation of cotton production (Hämmerle 2011). Furthermore, MMCFs are more environmentally sustainable than cotton owing to the reduced consumption of water and pesticides during productions. The advantages of MMCFs promoted further research on developing more sustainable fibre spinning processes (Schuster et al. 2004; Eichinger 2012). One emerging technology developed in recent years is the Ioncell process, which is currently in its pilot scale phase. In this process, fibres are spun from dissolving pulp-ionic liquid dope using the dry-jet wet spinning process. The Ioncell process is a Lyocell type process, but uses a thermally more stable solvent (Michud et al. 2014; Hummel et al. 2015; Sixta et al. 2015; Ma 2018). 
Most of the commercial MMCFs exhibit cellulose II structure after dissolution or mercerization and regeneration, such as Lyocell and viscose fibres (Jiang et al. 2012). Cellulose II structure provides better thermal stability compared to cellulose I because of more hydrogen bonds and shorter bond distance (Zugenmaier 2008; Maurer et al. 2013). Depending on the spinning technique, the crystalline size and the microfibril chain orientation are different. Lyocell type fibres demonstrated higher mechanical strength but lower elongation compared to viscose fibres due to the higher chain orientation along the longitudinal axis of the fibre (Hearle and Woodings 2001; Röder et al. 2013). This phenomenon is a result of stretching of the elastic dope in the air gap between the spinneret and the coagulation bath. The extensional stress created by the drawing of the filament leads to the orientation of the cellulose polymers in solutions along the fibre axis (Mortimer and Peguy 1996a; Fourne 1998).

Lyocell type fibres often have high fibrillation tendency in the wet, swollen state or under high mechanical abrasion conditions (Rohrer et al. 2001; Tomljenović and Čunko 2004; Zhang et al. 2005a; Goswami et al. 2009). The fibrillation occurs when crystalline orientation is high which leads to a weak lateral cohesion between crystalline sheets that eventually ease the delamination of the fibril structure on the axial of the fibre (Schurz and Lenz 1994; Mortimer and Péguy 1996b; Udomkichdecha et al. 2002). On the other hand, a viscose fibre or its derivative Modal fibre have lower fibrillation tendency due to their low molecular orientation. Furthermore, unlike viscose fibres that has a denser skin than the core, Lyocell fibres also have a more uniform structure throughout the whole cross section (from the surface to core) which is prone to swelling and fibrillation (Tomljenović and Čunko 2004). The textile produced with fibrillation effect will show a peach skin-like appearance that is problematic for weaving, dyeing and finishing (Bates et al. 2004; Goswami et al. 2009). However, fibrillation is proven to be an advantage for non-woven textile products (Mortimer and Péguy 1996b).

For the textile purpose, fibrillation must be minimized to maintain the quality of the product. The fibrillation can be reduced in several ways including changing the spinning parameters and crosslinking of the spun fibres during online spin finish using padding method followed by pre-drying and curing or the addition of chemicals in the spinning dope (Liu et al. 2016; Zhu and Liu 2018). The chemical crosslinking between the crosslinking agent and the cellulose takes place through the functionalization of the hydroxyl groups of the cellulose with the chemicals (Rojas and Azevedo 2011). Traditionally, crosslinking of the cellulose fibres for preventing the fibrillations or increasing the wrinkle recovery angle utilizes formaldehyde (Harifi and Montazer 2012). However, the use of formaldehyde should be limited or even avoided for health and sustainability reasons. New generation low toxic crosslinkers have been invented to replace formaldehyde. Lenzing AG has developed and commercially applied the triazine-based crosslinkers 1,3,5-triacryloyl-hexahydro-1,3,5-triazine (TAHT) and 2,4-dichloro-6-hydroxy-1,3,5-triazine for the reduction of fibrillation (Potter and Taylor 1998; Fuchs and Dobson 2009). Dimethyloldihydroxyethyleneurea (DMDHEU) has also been widely used for improving the final performance of the fibres on a commercial scale (Talukder and Manikandan 2016; Manian et al. 2017; Dhiman and Chakraborty 2017). To further reduce the toxicity and costs of the crosslinkers, benign crosslinkers based on dialdehydes, glyoxal and glutaraldehyde, are utilized for protecting the damaging of the fibres (Welch 1983; Choi et al. 1999; Stoyanoc et al. 2005; Yu et al. 2008). Furthermore, carboxylic acid-based crosslinkers such as citric acid and 1,2,3,4-butanetetra-carboxylic acid (BTCA) are thought to be sustainable approach for the crosslinking of cellulose (Yang et al. 1997; Yao et al. 2013; Aksoy and Genç 2015; Ye et al. 2015; Liu et al. 2016; Dhiman and Chakraborty 2017; Zhu and Liu 2018). Other researchers have also studied various sustainable or non-toxic crosslinkers to improve the quality of textiles, such as novel carboxylic acids (Maleic, Polymaleic and Succinic acid), phosphorus derivatives (phosphoric acid and Organophosphates), epichlorohydrin and silica derivatives (Yang et al. 2010; Rojas and Azevedo 2011; Udoetok et al. 2018).

As a relatively newly developed MMCF with the potential for commercialization, the Ioncell fibre has understandably not yet been characterized according to all textile-related aspects.

This work aims to develop a portable fibrillation testing method and evaluate the effect of crosslinking on the reduction of the fibrillation of the Ioncell fibre. Several fibrillation methods for MMFCs have been proposed previously including ball-bearing method, 
ultrasonication and high shear mixing method (Potter 1994; Mortimer and Péguy 1996b; Zhang et al. 2005a, b). However, the fibrillation determination procedures were not fully described. Thus, herein we propose a simple, time and energy saving scheme for a fibrillation test method. After successful generation of fibrillations and the quantification of the fibrils, the crosslinker TAHT was applied to the Ioncell fibres to investigate the influence of the crosslinking treatment conditions on the fibrillation index.

\section{Experimental methods}

\section{Materials}

Ioncell fibre was spun using methods described previously (Ma 2018). The raw material for standard Ioncell and high toughness Ioncell fibres are prehydrolyzed kraft birch pulp (Enocell, Stora Enso, Finland) and cold caustic treated pre-hydrolyzed kraft pine pulp (Buckeye V5S, Buckeye Technologies Inc., USA), respectively. Tencel and Modal staple fibre were provided by Lenzing AG. Viscose staple fibre was acquired from Sateri, China. The tensile properties of these fibres are listed in Table 1. The crosslinking agent 1,3,5-triacryloyl-hexahydro-1,3,5triazine (TAHT) was purchased from TCI, Japan.

Fibrillation tests

\section{Ball-bearing method}

$500 \mathrm{mg}$ of air-dried fibre was placed in a $100 \mathrm{ml} \mathrm{VWR}$ borosilicate 3.3 glass bottle containing 20 metal ball bearings (each metal ball has diameter of $0.5 \mathrm{~cm}$ and weight of $0.9 \mathrm{~g}$ ). $50 \mathrm{ml}$ of sodium carbonate solution
$(20 \mathrm{~g} / \mathrm{l})$ was added and the reactor was placed into the oven equipped with a tumbling rack. The glass reactor was tumbled end-over-end at a speed of $30 \mathrm{rpm}$ for $2 \mathrm{~h}$ at $80{ }^{\circ} \mathrm{C}$ for sufficient swelling of the fibres. The reactor was then removed from the oven and the ball bearings were separated utilizing a magnet. The remaining suspension was filtrated and the fibre was washed with an acetate buffer $(1 \mathrm{~mol} / \mathrm{l}$ acetic acid and $1 \mathrm{~mol} / \mathrm{l}$ sodium acetate) and water and then dried at $60{ }^{\circ} \mathrm{C}$ for $1 \mathrm{~h}$.

An alternative ball bearing method has also been applied but using a Linitest Original Banau machine. $500 \mathrm{mg}$ of fibres were placed in a metal pot with a volume of $500 \mathrm{ml}$. The treatment was carried out under similar conditions, however, in a water bath within the machine. The fibres were washed the same way as mentioned above.

\section{Blender method}

The creation of the microfibrils of the regenerated fibres was carried out using a Tefal Prep'line blender $(600 \mathrm{~W}) .500 \mathrm{mg}$ of air-dried regenerated fibre was cut into $\sim 5 \mathrm{~mm}$ short fibres and diluted with $500 \mathrm{ml}$ of water (or alkaline solution) in the blender. The fibre suspension was then mixed at varying conditions with the rotation speed of the blade of $60 \mathrm{~Hz}$ (approximately $3600 \mathrm{rpm}$ ). Subsequent to the mixing, the suspension was filtered and the fibre was washed and dried at $60{ }^{\circ} \mathrm{C}$ for 2 hours.

Assessment of fibrillation index

After fibrillation treatment, the dried fibres were cut into an even shorter length $(\sim 2 \mathrm{~mm})$ and diluted with water to a concentration of $1 \mathrm{~g} / \mathrm{l}$. The suspension was mixed well with $2 \%$ gelatin solution (as a fixation media) with a ratio of 1:1. Two drops of the fibre

Table 1 Tensile properties of the selected MMCFs for the fibrillation tests

\begin{tabular}{llllll}
\hline Fibre samples & Titer (dtex) & Dry tenacity (cN/tex) & Elongation (\%) & E-modulus (GPa) & Total orientation \\
\hline Standard Ioncell & 0.93 & 49.80 & 10.64 & 12.74 & 0.70 \\
High toughness Ioncell & 1.40 & 61.40 & 14.70 & 13.70 & 0.75 \\
Tencel & 1.27 & 36.78 & 15.58 & 8.00 & 0.62 \\
Viscose & 1.32 & 26.80 & 21.30 & - & - \\
Modal & 1.34 & 29.37 & 13.63 & 8.77 & - \\
\hline
\end{tabular}


suspension were dropped on to the objective glass from high in order to keep the fibrils open. A cover slide was placed and then the fibrillation of the fibres was examined using an optical microscope.

The microfibrils on the fibre surface was assessed using ImageJ software. The length of the fibre stem and the fibrils can be sketched by importing the microscopy images of the fibrillated fibre into the software and evaluated by means of the Simple neurite tracer function. For each fibre sample, 10 individual fibres were assessed to give an average value of the fibrillation index. The fibrillation index was calculated as follow:

$I_{f}=\frac{\sum_{L} l}{L}$

where $I_{f}$ being the fibrillation index, $l$ is the lengths of the fibrils over the length of the fibre $L$.

\section{Crosslinking}

The fibre samples were mixed first in TAHT alkaline solution (consists of $\mathrm{Na}_{2} \mathrm{CO}_{3}, \mathrm{Na}_{2} \mathrm{SO}_{4}$ or $\mathrm{NaOH}$ ) for $15 \mathrm{~min}$ in a $100 \mathrm{ml}$ VWR borosilicate 3.3 glass bottle at $80{ }^{\circ} \mathrm{C}$ in an oven equipped with tumbling rack. The treated fibre was either used as it is or nipped and subjected to drying at $120{ }^{\circ} \mathrm{C}$ and steaming at $100{ }^{\circ} \mathrm{C}$ for 2 and 7 min respectively. In case the mixing stage was skipped, the fibre was only dipped into the crosslinking solution prior to drying or steaming. Following the treatment, the fibres were filtered and washed with $1 \%$ acetic acid and water until the filtrate is neutral.

\section{Elemental analysis}

The elemental analysis was conducted using a Perkin Elmer 2400 element analyzer under helium environment. The weight percentage of oxygen content in the samples was obtained by deducting the weight percentages of carbon, hydrogen, nitrogen and sulphur from $100 \mathrm{wt} \%$. Sulphanilamide was used as standard.

\section{Scanning electron microscopy (SEM)}

SEM imaging was performed by using a Zeiss Sigma VP with variable pressure. The fibres were attached onto the conductive support and sputtered with gold for $5 \mathrm{~nm}$ to ensure electric conductivity. The images were taken at $3 \mathrm{kV}$ operating voltage.

Tensile properties

Tensile properties of the untreated and treated fibres were analyzed using the automatic single-fibre tester Favigraph instrument (Textechno H. Stein GmbH \& Co, Germany). Tenacity and elongation of the fibres were measured in conditioned state. Twenty fibres from each sample were tested at $23{ }^{\circ} \mathrm{C}$ and $60 \% \mathrm{RH}$. The gauge length was $20 \mathrm{~mm}$ and speed $20 \mathrm{~mm} / \mathrm{min}$. The elastic modulus was calculated from the slope of the stress-strain curve of the fibre based on ASTM D2256 standard using MATLAB.

\section{Results and discussion}

Fibrillation tests

The fibrillation tests were conducted by employing wet abrasion in different conditions. Initial tests were done by adopting ball-bearing method as described by Zhang et al. (2005a, b), but using a modified device. The fibrillation tests were first carried out by using a glass bottle in which $500 \mathrm{mg}$ Ioncell or Tencel fibres were immersed with $50 \mathrm{ml}$ of $20 \mathrm{~g} / 1 \mathrm{Na}_{2} \mathrm{CO}_{3}(\mathrm{pH}$ 11.3). Twenty metal spheres were transferred to the bottle and then tumbled in an oven at a temperature of $80{ }^{\circ} \mathrm{C}$ for 2 hours. However, the fibrillation tendency was not pronounced and uneven distributed on both Ioncell and Tencel fibres; part of the fibres showed no fibrillations. On a closer observation of the experiment, we have confirmed that the metal balls tended to slip from the fibres during the mixing, which led to less wet abrasion. The ratio between the treatment solution and the volume of the container was too high. As a result, the fibres floated during the treatment and had little contact with the metal balls. Figure 1 illustrates the Ioncell fibre after the first ball bearing fibrillation treatment method in the glass reactor.

In order to improve the wet abrasion of the fibres through the metal balls, this ball-bearing fibrillation method was modified by using a Linitest machine to which a metal pot with a volume of $550 \mathrm{ml}$ was attached. The same amount of fibre $(500 \mathrm{mg})$, alkaline solutions $\left(50 \mathrm{ml}\right.$ of $20 \mathrm{~g} / \mathrm{l} \mathrm{Na} \mathrm{CO}_{3}$ ) and metal balls that used in the glass reactor were added in the metal 


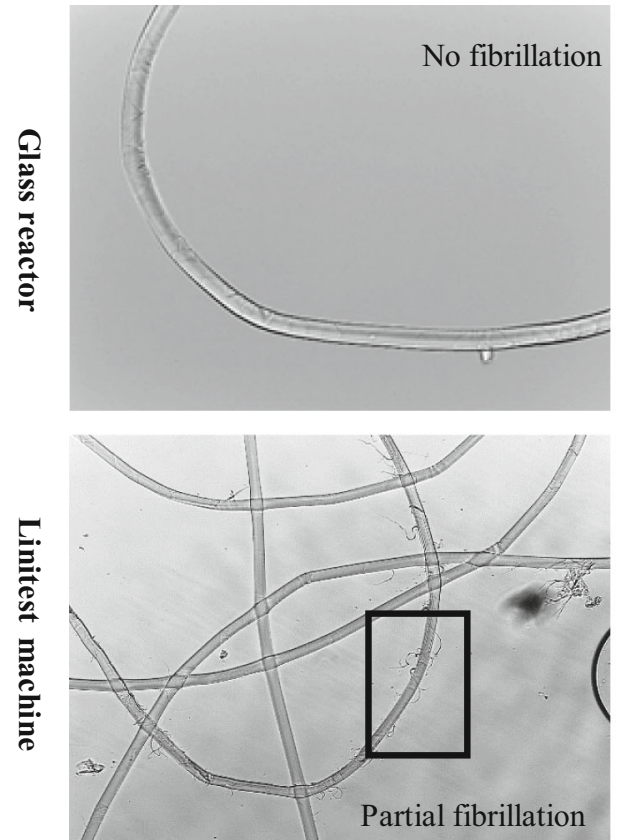

Fig. 1 Microscopy images of fibrillated standard Ioncell fibres after ball-bearing treatment in the glass bottle and Linitest machine. Upper row: standard Ioncell fibre treated in glass

pot. The metal pot was rotated in a water bath inside the Linitest machine for $2 \mathrm{~h}$ at controlled temperature of $80{ }^{\circ} \mathrm{C}$. Surprisingly, neither Ioncell nor Tencel fibres showed significant fibrillation. The unexpected results led us to the conclusion that the fibres were not well swollen during the treatment. As previously reported by Manian et al. (2017), swelling is a necessary factor for the fibres to form fibrils when using the ball-bearing method. Another possible explanation for the unsatisfactory results could be the limitation of the treatment device, which cannot provide sufficient abrasion on the fibres. The fibres tended to form a lump in the aqueous medium. Then the metal balls did not slide along the length of the fibres with each revolution, but simply dropped to the other side of the vessel, after which the much lighter fibre lump just fell on top of them. In addition, the formation of the fibre agglomerate prevents the fibres inside the lump from any abrasion by passing metal balls, resulting in unevenly fibrillated fibres.

To improve the swelling of the fibres, the $\mathrm{pH}$ of the solution was then adjusted by replacing $\mathrm{Na}_{2} \mathrm{CO}_{3}$ with $\mathrm{NaOH}$. In addition, the number of ball bearings was increased from 20 to 50 to increase the abrasive forces acting on the fibres. With a solution containing $4 \mathrm{~g} / \mathrm{l}$
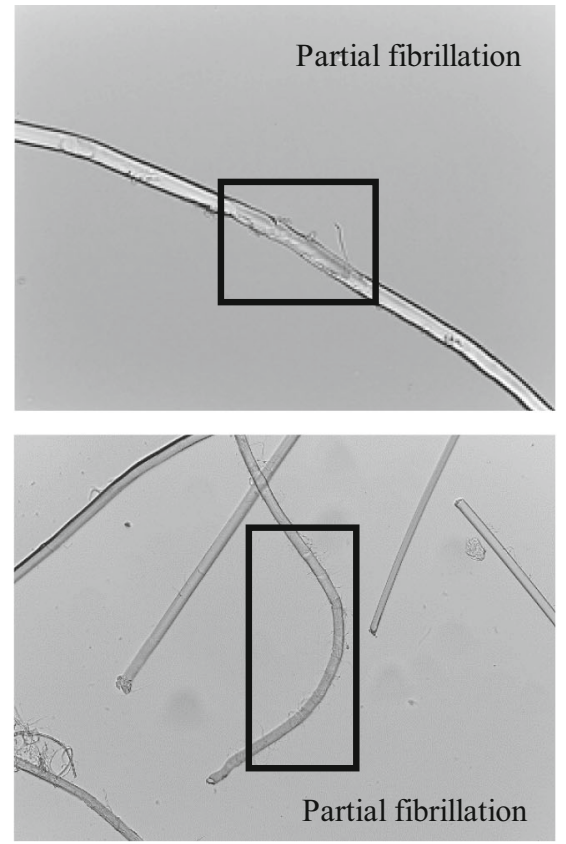

reactor for $2 \mathrm{~h}$ at $80{ }^{\circ} \mathrm{C}$ in $20 \mathrm{~g} / 1 \mathrm{Na}_{2} \mathrm{CO}_{3}$ solution. Bottom row: standard Ioncell fibre treated in Linitest machine for $2 \mathrm{~h}$ at $80{ }^{\circ} \mathrm{C}$ in $20 \mathrm{~g} / 1 \mathrm{NaOH}$ solution

$\mathrm{NaOH}$ ( $\mathrm{pH}$ 12.6), still no significant number of fibrils was delaminated from the fibre surfaces. Ultimately, the $\mathrm{pH}$ of the solution was increased to 14.1 by using a $20 \mathrm{~g} / \mathrm{l} \mathrm{NaOH}$ solution. At such highly alkaline condition, part of the fibres were swollen, and a large amount of fibrils could be identified on the fibre surface. On the other hand, fibres with little or no swelling showed hardly any fibrillation. This observation confirmed the influence of the fibre swelling on the fibrillation tendency of the MMCFs. Despite visible fibrillation of the swollen fibres, it was still not possible to determine a representative fibrillation index due to the uneven fibrillation of the individual fibres (see Fig. 1). In summary, the ball bearing method failed to create sufficient fibrillation and did not yield satisfying results despite of high time and energy consumption.

Therefore, an alternative fibrillation method was developed. The fibrillation tests of the standard and high toughness Ioncell fibres, Tencel, viscose and modal fibres were established by means of wet abrasion using a laboratory blender. The fibrillation was then assessed using an optical microscope and the index was calculated by the ratio of the sum of the fibril length and the length of the fibre. It should be 
noted that when preparing the fibre suspension for microscopy imaging, the gelatin solution had to be added. The addition of the gelatin solution kept the fibrils open on the fibre surface and prevented the fibre from floating freely. Consequently, reliable images could be taken and processed for evaluation.

In order to evaluate the reliability and reproducibility of the blender method, both standard Ioncell and Tencel fibres were treated in the blender 5 times in water at ambient conditions for $5 \mathrm{~min}$. Figure 2. illustrates the reproducibility of these fibrillation tests. Fibres treated in the blender showed clear micro fibrils along the fibre surface (as shown in Figs. 3 and 4. The fibrillation indexes differed slightly between each fibrillation tests for both standard Ioncell and Tencel fibres. However, the standard deviation of the fibrillation index for each individual tests are higher, indicating a certain intrinsic scattering among each fibre sample. Nevertheless, the average value of the fibrillation index was still representative for the fibrillation tendency of the sample, especially when comparing with other fibre types or different treatment condition. Those samples showed a clearly differ fibrillation index. The absolute fibrillation index may vary when using a different blender or at different conditions, however, the methods provides reliable results in comparative studies as presented here.

The fibrillation tests were carried out under different conditions, including treatment media, temperature and duration. Figure 3 illustrates the difference between the fibrillations index of Tencel, standard and

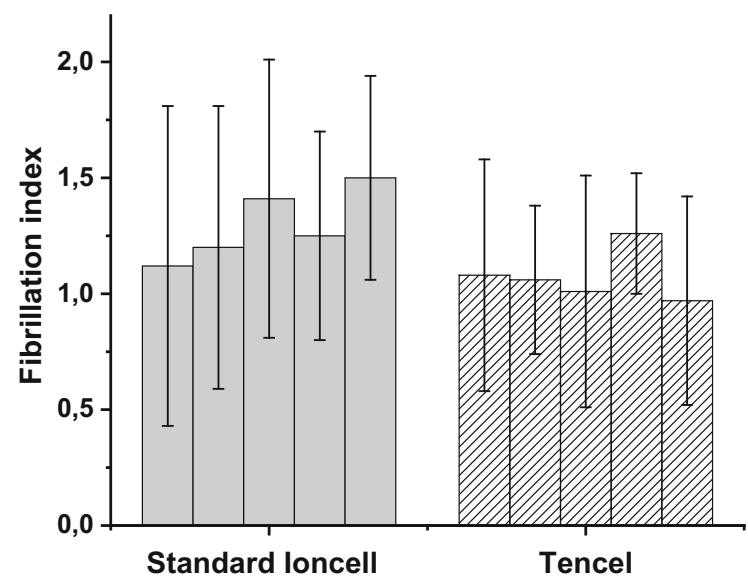

Fig. 2 Reproducibility of fibrillation tests using the blender. The fibres were treated in the blender at ambient conditions in water for $5 \mathrm{~min}$ high toughness Ioncell fibres and the microscopic images of the fibrillated fibres, respectively. All Ioncell and Tencel fibres tended to fibrillate under wet abrasion using the blender. The longer the treatment produced the greater the number of fibrils along the fibre surface (Potter and Dobson 1995). However, no obvious fibrillation could be observed on the viscose and modal fibres. The orientation of the cellulose molecules was the most dominating factor governing the fibrillation of the regenerated fibre. Standard Ioncell and Tencel fibres demonstrated similar fibrillation results. The high toughness Ioncell fibres showed a significantly higher fibrillation index compared to standard Ioncell fibre and Tencel, owing to its very high cellulose orientation. The well-aligned crystalline regions have a reduced lateral cohesion, thus, lead to enhanced fibrillation of the fibres under mechanical stress (Mortimer and Péguy 1996b; Lim et al. 2003). Moreover, for high toughness Ioncell fibre, the reduction in the hemicellulose content, the relatively uniformly long molecular chains and the less amorphous region contributed to a higher orientation of the fibre and thus promote the delamination of its fibre surface. As expected, the viscose fibres had a negligible fibrillation index even under such high shear force due to their lower orientation, the formed crystallite clusters and denser surfaces (Schurz and Lenz 1994).

Figure 4 presents the SEM images of the fibrillated fibres using a laboratory blender for 5 and $10 \mathrm{~min}$ in water. It could be clearly seen that after a 5 min treatment in water, the surface of the fibre remains relatively smooth and shows only a few individual fibrils. Due to the high shear force applied in the mixer, a large piece of skin was peeled off the fibre. As the treatment intensity increased, more fibrils were formed, which are generally longer than with lower treatment intensity. The skin of the fibre was deteriorated at this higher wet abrasion due to a large amount of the delaminated fibrils.

The influence of the treatment temperature and alkalinity of the solution media on the fibrillation index of the fibres was assessed by mixing the Ioncell and Tencel fibres in the blender for 5 min with $20 \mathrm{~g} / \mathrm{l}$ $\mathrm{Na}_{2} \mathrm{CO}_{3}$ and $80{ }^{\circ} \mathrm{C}$. As shown in Fig. 5, both higher temperature and the alkaline environment increased the fibrillation index of the fibres. The alkalinity seemed to provide more severe delamination of the fibre than that in the high-temperature treatment. 

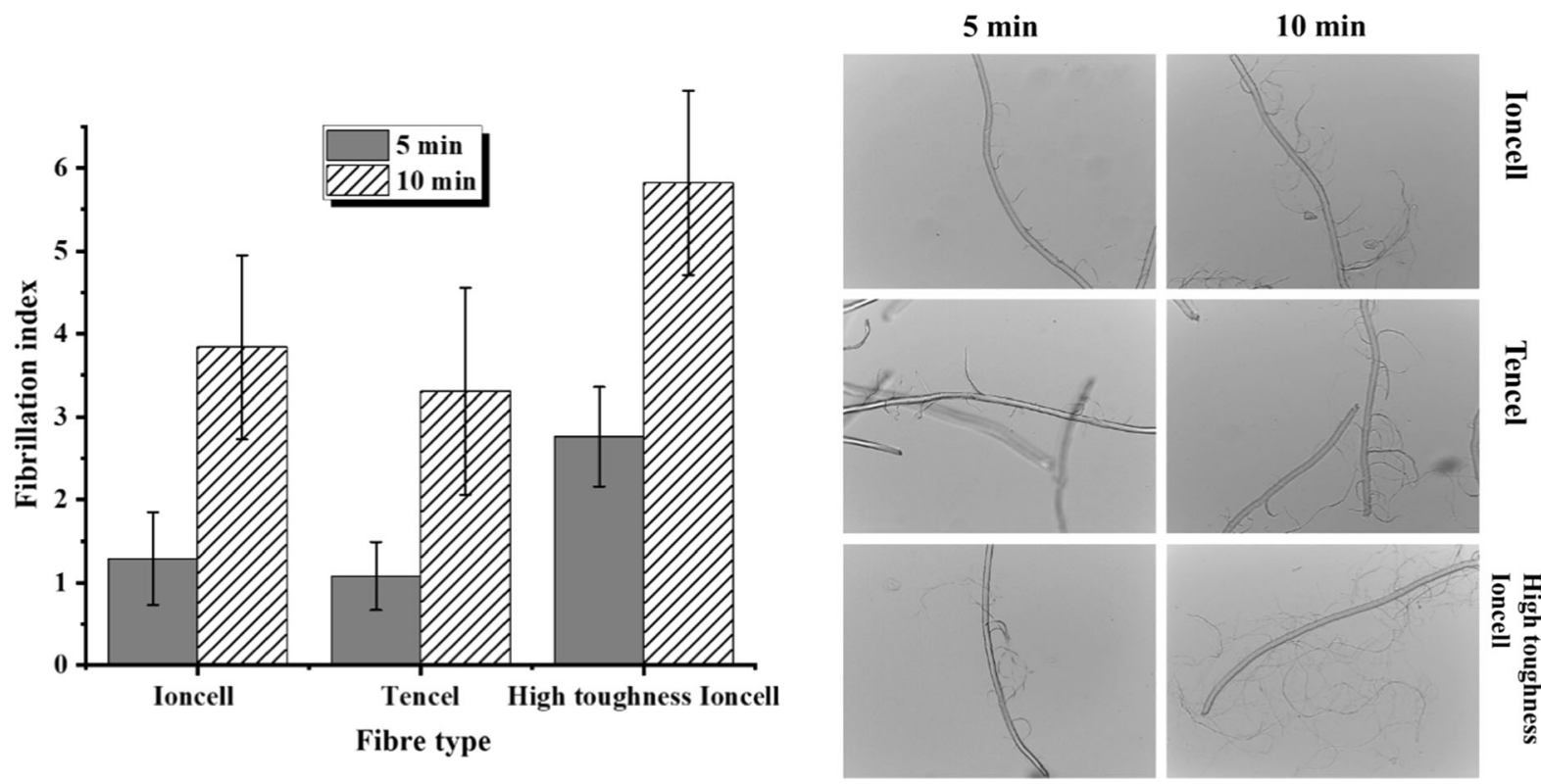

Fig. 3 Influence of the wet abrasion treatment times on the fibrillation index of fibre and microscope images of the fibrillated fibres
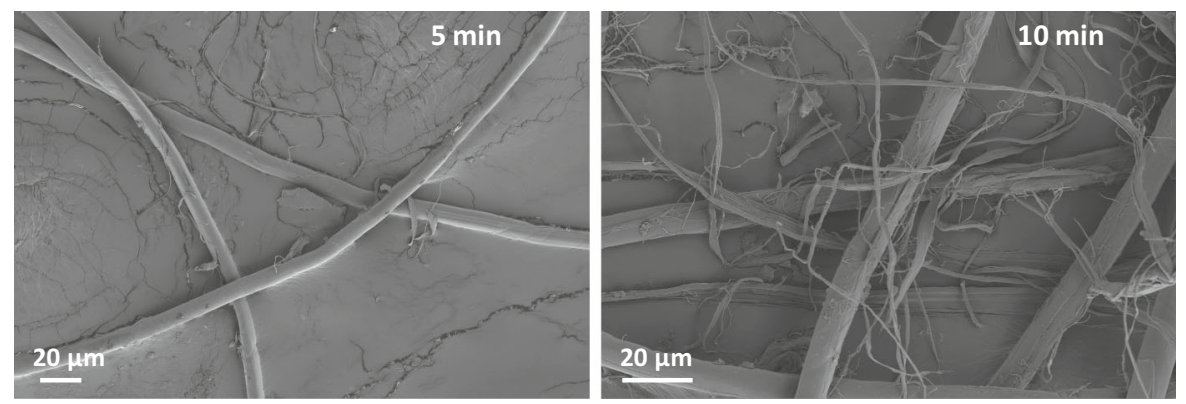

Fig. 4 SEM images of the fibrillated Ioncell fibres in water for 5 and 10 min using the blender

When the fibres were subjected to fibrillation treatment in alkaline solution ( $\mathrm{pH} 11.3$ ), the fibrillation index was more than twice that of the reference treatment. The combination of both higher temperature and alkaline media resulted in a significant increase in the fibrillation tendency of the fibres. The findings are consistent with previous work done by Zhang et al. in which it has been claimed that the fibrillation tendency of the Lyocell fibres can be enhanced by the prolonged treatment time, higher alkalinity and temperature of the treatment media (Zhang et al. 2005a, b). The higher temperature and alkaline concentration enhanced the swelling of the fibres, resulting in fibres with weak hydrogen bonds between lateral crystallites that led to severe fibrillation. The standard deviation of the fibrillation index was exceptionally high, especially when the fibrillation was strong. This can be attributed to the inevitable unevenness of the force exerted on the fibres that resulted in a variation of the fibrillation index.

Surprisingly, even under alkaline $\left(20 \mathrm{~g} / \mathrm{l} \mathrm{Na}_{2} \mathrm{CO}_{3}\right)$ and high-temperature conditions, fibrillations on the viscose and modal fibres were still extremely low and unevenly distributed on the fibres. Especially for viscose fibre, the fibrils created on the fibre surface were very small, which can only be observed with high magnification under the microscope (Fig. 6). This observation confirms that the low orientation of the cellulose molecules mitigates the fibrillation on the fibre surface. Furthermore, the very short fibrils that 

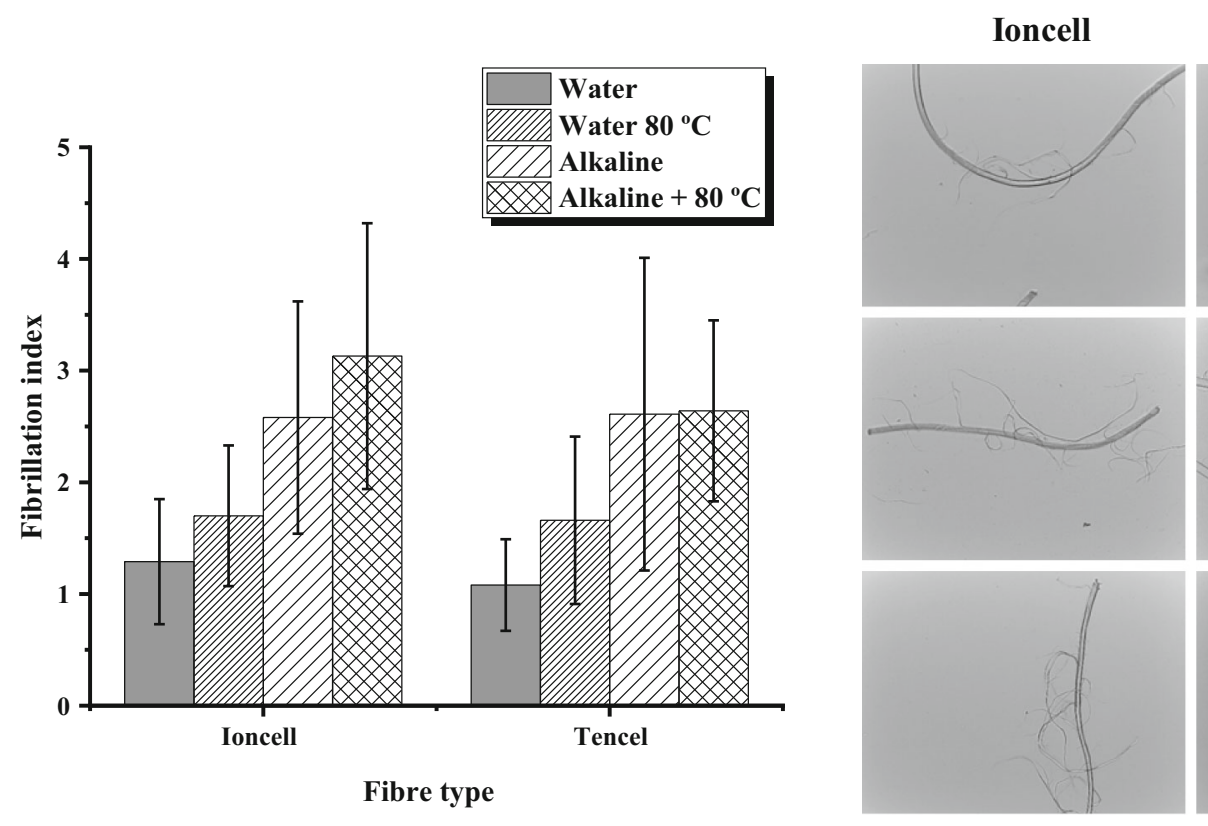

\section{Tencel}

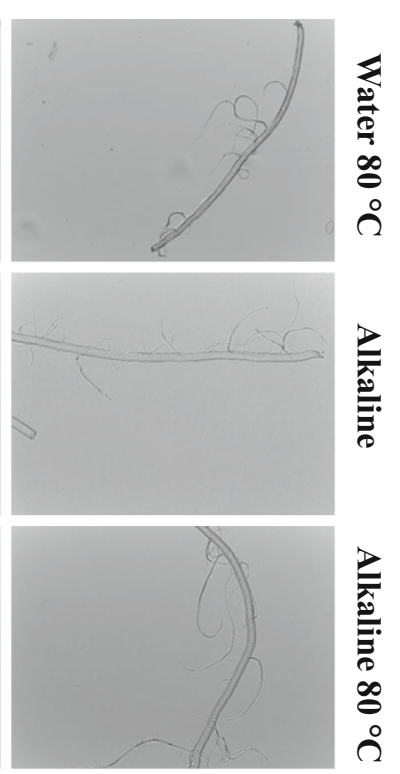

Fig. 5 Effect of the treatment temperature and alkalinity of the media on the fibrillation tendency of the fibres

Fig. 6 Fibrillation of viscose and modal fibres in water and alkaline conditions

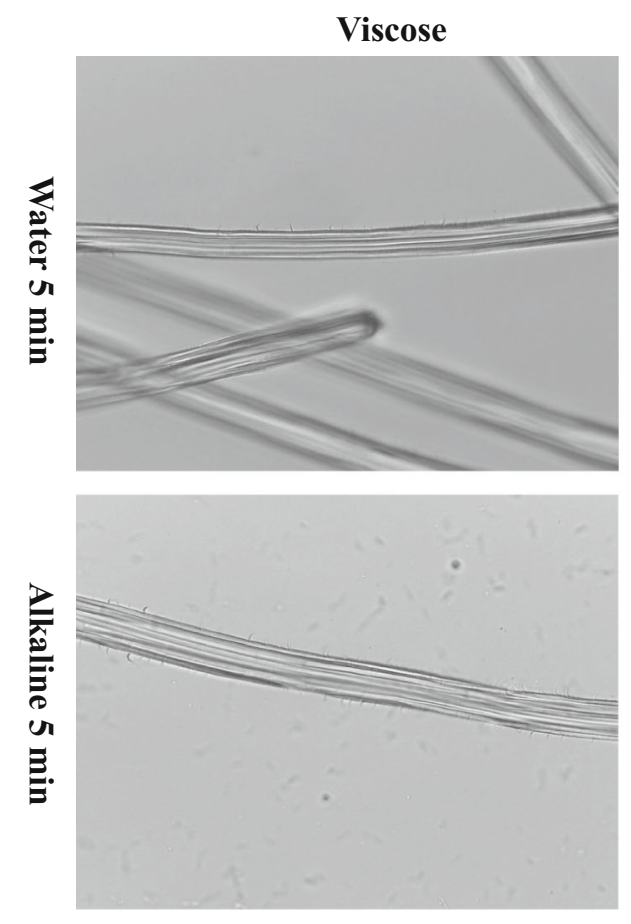

\section{Modal}
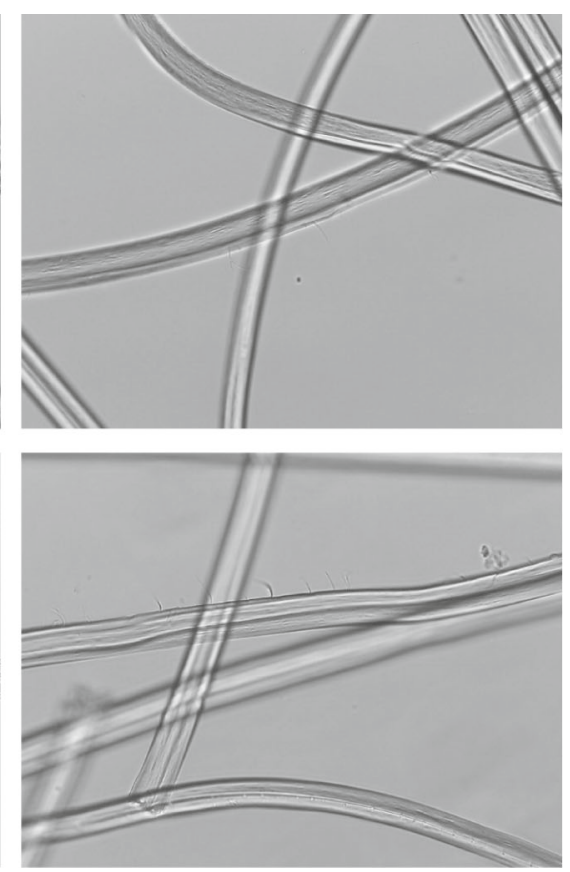

appeared on the fibre can also be explained by their denser skin and possible shorter cellulose chain.
Crosslinking with 1,3,5-triacryloyl-hexahydro1,3,5-triazine (TAHT)

TAHT has been widely used as cellulose crosslinker to successfully reduce the fibrillation in Lenzing AG and 
Courtaulds Ltd for Tencel fibres (Rohrer et al. 2001). This triazine-based crosslinker contains three acrylamido groups that react with the hydroxyl groups on the cellulose in alkaline condition with a preferred $\mathrm{pH}$ of 11.5-12.5. The crosslinker may undergo hydrolysis if the $\mathrm{pH}$ is too high or the crosslinker solution has been stored for a long time. Thus, the $\mathrm{pH}$ of the alkaline crosslinking solution must be carefully controlled and the reaction started as soon as the solution is prepared (Potter 1994; Lewis et al. 2002).

The influence of the crosslinker concentration was studied at a constant solution to fibre ratio of 12.5:1 to keep the water and chemical consumption as low as possible. Ioncell and Tencel fibres demonstrated more pronounced fibrillation during the wet abrasion tests and were selected for crosslinking. The fibres were treated in various steps to determine the most efficient method for fixing the crosslinker on the fibres, including mixing of fibres in the crosslinking solution for $15 \mathrm{~min}$ at $80{ }^{\circ} \mathrm{C}$, drying at $120{ }^{\circ} \mathrm{C}$ for $2 \mathrm{~min}$ and steam with saturated steam at $100{ }^{\circ} \mathrm{C}$ for $7 \mathrm{~min}$ (including 2 min heating-up). It was found that while mixing the fibres in the crosslinking solution, the dissolution of TAHT in the alkaline solution $(\mathrm{NaOH}$ $10 \mathrm{~g} / \mathrm{l})$ and the crosslinking reaction took place at the same time. Complete dissolution of the TAHT in the solution took approximately $5 \mathrm{~min}$. The fibrillation index of the fibres was determined using the blender fibrillation method described in the previous section.

Table 2 depicts the effect of the treatment steps and freshness of the crosslinker solution on the fibrillation tendency of Tencel and Ioncell fibres. The results showed that the freshness of the solution has a significant influence on the fibrillation tendency of the fibres when using a constant TAHT concentration of $10 \mathrm{~g} / \mathrm{l}$ in the solution ( $12.5 \mathrm{wt} \%$ on fibre). The crosslinker solution stored for one day has already lost most of its ability to react with the hydroxyl groups of the cellulose. The hydrolysis of the acrylamido groups in alkaline solution prevented the bridging of the crosslinker with cellulose. The fibrillation of the Tencel fibres crosslinked using an old solution led to a fibrillation index of 0.76 which was only slightly lower than the value that without crosslinking (1.08). While using fresh solutions in which the TAHT dissolution and crosslinking with cellulose occurred at the same time, the treated fibres demonstrated high resistance to the wet abrasion. The well-crosslinked fibres showed negligible fibrillation tendency.
The treatment of fibres at high temperature are crucial steps in order to fix the crosslinker onto the cellulose. Mixing of the fibres in the crosslinker solution at $80^{\circ} \mathrm{C}$ is an utmost important stage for crosslinker fixation. The fibres underwent treatment stages including mixing indicated promising reduction of the fibrillation index. According to Table 2, a single mixing treatment could already prevent the fibres from fibrillation, which indicated that the crosslinkers reacted already with the cellulose hydroxyl groups. The additional treatment step of drying and steaming did not further improve the fibrillation. Without the mixing stage, the steaming treatment or drying combined with steaming treatment revealed slightly higher fibrillation index. However, drying and steaming can have a positive effect on the water retention and wrinkle recovery of the textile ( $\mathrm{Li}$ et al. 2019). Nevertheless, the current work only focuses on the fibrillation tendency of the fibres. Thus, we have chosen to use the single mixing treatment for the further assessment of the crosslinking tests.

The fibrillation tendency of the crosslinked fibres is closely related to the concentration of the crosslinking solution. Figure 7 illustrates the trend of the fibrillation index of Tencel and Ioncell at varying TAHT concentration (on fibre) in the reagent solution. The fibrillation index of the crosslinked Tencel and Ioncell fibres already decreased drastically when the amount of TAHT in the solution was $2.5 \mathrm{wt} \%$ on fibres. The fibrillation index of the fibres was nearly diminished to 0 with a TAHT amount of $12.5 \mathrm{wt} \%$ on fibre. Further addition of TAHT did not alter the results. When the duration of the fibrillation treatment was increased from 5 to $10 \mathrm{~min}$, the fibrillation index of the crosslinked fibres was slightly higher than that of 5 min due to the intense shearing force applied to the fibres. However, the reduction of the fibrillation was also significant, when the TAHT concentration was increased. The fibrillation tendency of crosslinked standard Ioncell fibres was higher compared to that of Tencel fibres owing to its higher degree of orientation.

Despite the determination of the fibrillation index reflected by the TAHT concentration, it is also worthwhile to evaluate the actual amount of the TAHT fixed on the cellulose fibres. Table 3 lists the factors that influence the amount of the TAHT attached to the fibres. The results indicated that both treatment methods and TAHT concentration in the solution could affect the crosslinker fixation. It is 
Table 2 Influence of the freshness of the reagent solution and treatment steps on the fibrillation of the Tencel and Ioncell fibres

\begin{tabular}{llll}
\hline Fibre & Treatment* & Solution type*** & Fibrillation index \\
\hline Tencel reference & - & - & 1,08 \\
Tencel & $\mathrm{H}, \mathrm{D}, \mathrm{S}$ & Old & 0.76 \\
Tencel & $\mathrm{H}, \mathrm{D}, \mathrm{S}$ & Fresh & 0.05 \\
Tencel & $\mathrm{H}, \mathrm{S}$ & Fresh & 0.004 \\
Tencel & $\mathrm{S} * *$ & Fresh & 0.17 \\
Tencel & $\mathrm{H}$ & Fresh & 0.06 \\
Ioncell reference & - & - & 1.29 \\
Ioncell & $\mathrm{H}, \mathrm{D}, \mathrm{S}$ & Fresh & 0.07 \\
Ioncell & $\mathrm{D}, \mathrm{S} * *$ & Fresh & 0.18 \\
Ioncell & $\mathrm{H}$ & Fresh & 0.02 \\
\hline
\end{tabular}

TAHT concentration in solution was set to $10 \mathrm{~g} / \mathrm{l}(12.5 \mathrm{wt} \%$ on fibre)

$* \mathrm{H}=$ Mixing of fibre in crosslinker solution at $80{ }^{\circ} \mathrm{C}$ for $15 \mathrm{~min} . \mathrm{D}=$ Drying the crosslinked fibre at $120{ }^{\circ} \mathrm{C}$ for $2 \mathrm{~min} . \mathrm{S}=\mathrm{Steaming}$ of the crosslinked fibre at $100{ }^{\circ} \mathrm{C}$ for $7 \mathrm{~min}$

**Without mixing stage $(\mathrm{H})$, the fibre was dipped and nipped two times in the crosslinker solution at room temperature prior to the drying and steaming

***Fresh solution is being prepared in situ during the crosslinking treatment. Old solution is prepared one day prior to the crosslinking treatment

Table 3 Effect of treatment methods and crosslinker concentration in solution on the crosslinker attached on the fibres

\begin{tabular}{|c|c|c|c|c|}
\hline Fibre & Treatment & TAHT conc. in solution $\mathrm{g} / \mathrm{l}$ & TAHT $\%$ o.w.f.*** & TAHT attached on fibre wt $\%$ \\
\hline Tencel & $\mathrm{H}, \mathrm{D}, \mathrm{S}$ & 40 & 50 & 7.70 \\
\hline Tencel & $\mathrm{H}, \mathrm{D}, \mathrm{S}$ & 20 & 25 & 6.90 \\
\hline Tencel & $\mathrm{H}, \mathrm{D}, \mathrm{S}$ & 10 & 12.5 & $0 *$ \\
\hline Tencel & $\mathrm{H}, \mathrm{D}, \mathrm{S}$ & 2 & 2.5 & $0 *$ \\
\hline Ioncell & $\mathrm{H}$ & 10 & 12.5 & 2.29 \\
\hline Ioncell & $\mathrm{H}$ & 7 & 8.75 & 1.37 \\
\hline Ioncell & $\mathrm{H}$ & 5 & 6.25 & 1.10 \\
\hline Ioncell & $\mathrm{H}$ & 2 & 2.5 & 1.42 \\
\hline Ioncell & $\mathrm{D}, \mathrm{S}^{* *}$ & 10 & 12.5 & 0.16 \\
\hline
\end{tabular}

*One day-old crosslinker solution applied

**Prior to treatment, the fibre was only dipped in the crosslinking solution and nipped before drying stage

$* * *$ On the weight of fibre

important to include the mixing stage in order to enhance the fixation of the crosslinker on the fibres; simple dipping of the fibres in the crosslinking solution followed by drying and steaming could not promote the reaction between the crosslinker and the cellulose hydroxyl groups. Further, an aged solution containing degraded TAHT was not able to react with the hydroxyl groups.
The amount of TAHT fixed on the fibres reduced as the crosslinker concentration dropped. However, the fixation rate seemed to be the opposite. A $50 \mathrm{wt} \%$ (on fibre) TAHT reagent solution resulted in $7.7 \mathrm{wt} \%$ of TAHT being linked to the fibres. On the other hand, when the TAHT concentration was lowered to $2.5 \mathrm{wt} \%$ on fibre, more than half of the available TAHT was found attached to the fibres. The 


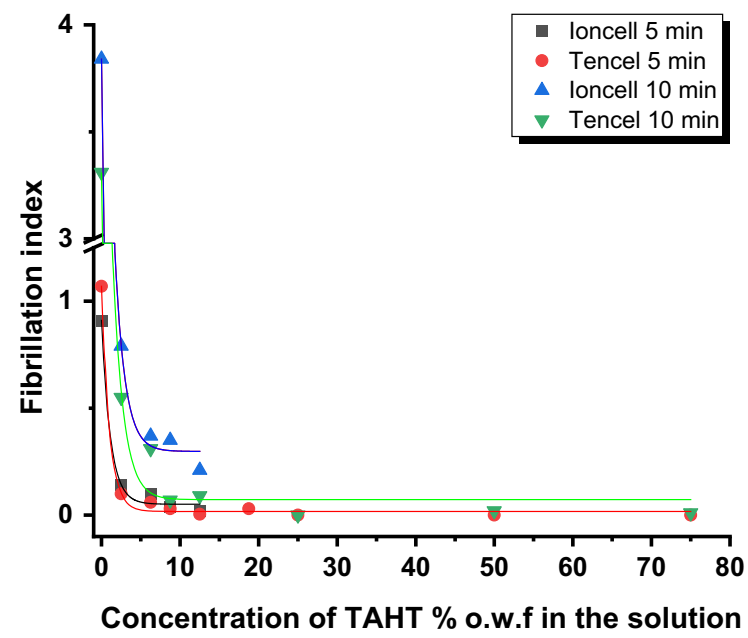

Fig. 7 Influence of the TAHT concentration in the crosslinking solution on the fibrillation tendency of the Tencel and Ioncell fibres

fibrillation tendency of the crosslinked Ioncell fibres as function of the attached crosslinker followed the same trend as the TAHT solution concentration dependency. As shown in Fig. 8, the fibrillation index of the crosslinked fibres can be reduced to a very low level even if only $1.1 \mathrm{wt} \%$ of TAHT was crosslinked on the fibre.

The crosslinking of the TAHT with cellulose has a pivot effect on the tensile properties of the fibres. Figure 9 presents the tensile properties of the Ioncell fibres as the function of the TAHT concentration. Gradually increasing the amount of TAHT during the crosslinking treatment reduced the mechanical performance of the fibres. While $2.5 \mathrm{wt} \%$ of TAHT was added, the crosslinked Ioncell fibres lost approximately $10 \%$ of its original strength. A $16 \%$ strength loss was observed when the Ioncell fibre was treated with a $12.5 \mathrm{wt} \%$ TAHT solution. The elongation of the fibres was also deteriorated due to the crosslinking reaction. The reduction in mechanical performance might also result from the alkaline degradation of the cellulose during the crosslinking. Moreover, the crosslinking of the cellulose chains restricts their elasticity, which makes the fibres stiffer and more brittle (Patil and Netravali 2019). Despite the loss in the tensile properties, the strength of the fibre was still comparable to the untreated Tencel fibres.

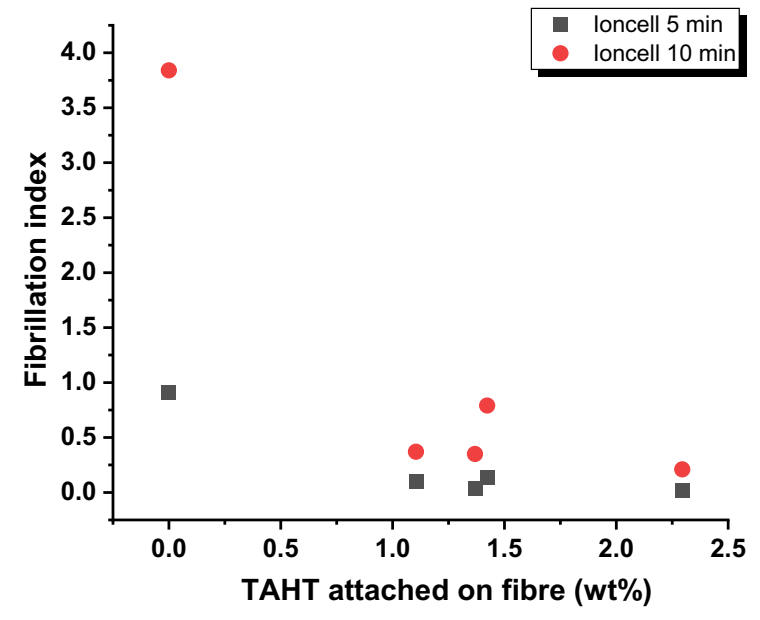

Fig. 8 Influence of TAHT attached on the fibres on the fibrillation of crosslinked Ioncell fibres

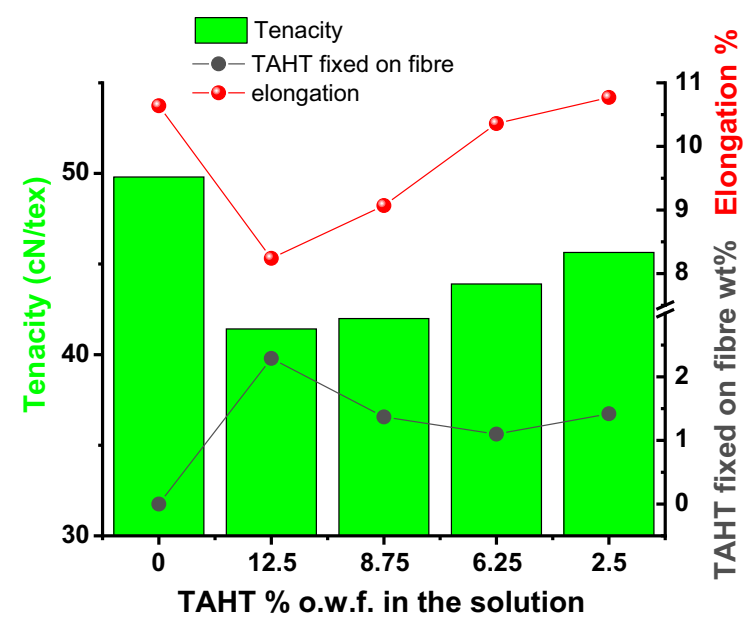

Fig. 9 The effect of the crosslinking on the tensile properties of the Ioncell fibres

\section{Conclusions}

In this study, we provide a routine method for the determination of the fibrillation index of the Lyocelltype fibres using a laboratory blender. The method offers a more time and energy-saving route to create micro-fibrils on the fibre surface compared to the earlier proposed ball-bearing methods. The fibrillation tests based on this method confirmed the effect of the 
fibre orientation and the conditions of treatment media (time, temperature and alkalinity) on the fibrillation index of the fibres. The fibres with a higher orientation showed a higher fibrillation tendency. Due to the low alignment of the cellulose molecules and denser skin, viscose and modal fibres demonstrated a very low tendency of fibrillation. Increasing the treatment intensity by rising the treatment time, temperature and alkalinity significantly enhanced the fibrillation index of the Tencel and Ioncell fibres.

The blender method proved to be a reliable tool for comparative studies of the fibrillation tendency of different fibres and was used to investigate the effect of the crosslinking Tencel and Ioncell fibres. Crosslinking of Ioncell fibres with TAHT has been successfully performed to reduce fibrillation. The stabilization against fibrillation relied strongly on the TAHT concentration in the treatment solution. Since THAT underwent fast degradation it is important to use a fresh reagent solution, ideally prepared in situ in the presence of the fibres. The fibrillation tendency reduced significantly already at a concentration of $2.5 \mathrm{wt} \%$ (on fibre) of TAHT (1.42 wt \% were actually attached on the fibre). The fibrillation index seemed to be minimized at a TAHT concentration of $12.5 \mathrm{wt} \%$ (on fibre) (only $2.29 \mathrm{wt} \%$ were fixed on fibre) was utilized. Mixing the fibres with the crosslinking solution at $80{ }^{\circ} \mathrm{C}$ for $15 \mathrm{~min}$ was the most crucial step for the fixation of the crosslinker to the fibres. Without this stage, subsequent high-temperature predrying and steaming could not promote efficiently the reaction of TAHT with the cellulose hydroxyl group. After crosslinking, the mechanical properties of the fibres were slightly reduced possibly owing to the degradation of the cellulose chains in the alkaline medium. Reduction in the elasticity was also a direct result of the crosslinking. However, the strength of the crosslinked Ioncell fibres was still comparable to the untreated Tencel fibres.

Acknowledgments This project is financially supported by Sateri International, Singapore. The author would like to thank Ms. Kaisa Hytti for performing the elemental analysis.

Funding Open access funding provided by Aalto University.

Open Access This article is licensed under a Creative Commons Attribution 4.0 International License, which permits use, sharing, adaptation, distribution and reproduction in any medium or format, as long as you give appropriate credit to the original author(s) and the source, provide a link to the Creative Commons licence, and indicate if changes were made. The images or other third party material in this article are included in the article's Creative Commons licence, unless indicated otherwise in a credit line to the material. If material is not included in the article's Creative Commons licence and your intended use is not permitted by statutory regulation or exceeds the permitted use, you will need to obtain permission directly from the copyright holder. To view a copy of this licence, visit http://creativecommons.org/licenses/by/4.0/.

\section{References}

Aksoy SA, Genç E (2015) Functionalization of cotton fabrics by esterification cross-linking with 1,2,3,4-butanetetracarboxylic acid (BTCA). Cellul Chem Technol 49:405-413

Bates I, Maudru E, Phillips DAS et al (2004) Cross-linking agents for the protection of lyocell against fibrillation: synthesis, application and technical assessment of 2,4-diacrylamidobenzenesulphonic acid. Color Technol 120:293-300. https://doi.org/10.1111/j.1478-4408.2004. tb00233.x

Choi HM, Kim JH, Shin S (1999) Characterization of cotton fabrics treated with glyoxal and glutaraldehyde. J Appl Polym Sci 73:2691-2699. https://doi.org/10.1002/ (SICI)1097-4628(19990923)73:13<2691::AIDAPP17>3.0.CO;2-T

Dhiman G, Chakraborty JN (2017) Assessment of durable press performance of cotton finished with modified DMDHEU and citric acid. Fash Text 4:1-18. https://doi.org/10.1186/ s40691-017-0104-2

Eichinger D (2012) A vision of the world of cellulosic fibers in 2020. Lenzinger ber 90:4-10

Fourne F (1998) Synthetic fibers. Hanser/Gardner Publications, Ohio

Fuchs H, Dobson P (2009) Cellulose fiber and method for the production thereof. WO 2009/155624 A1

Goswami P, Blackburn RS, El-Dessouky HM et al (2009) Effect of sodium hydroxide pre-treatment on the optical and structural properties of lyocell. Eur Polym J 45:455-465. https://doi.org/10.1016/j.eurpolymj.2008.10.030

Hämmerle FM (2011) The cellulose gap (the future of cellulose fibres). Lenzinger Ber 89:12-21

Harifi T, Montazer M (2012) Past, present and future prospects of cotton cross-linking: new insight into nano particles. Carbohydr Polym 88:1125-1140. https://doi.org/10.1016/ j.carbpol.2012.02.017

Hearle JWS, Woodings C (2001) Fibres related to cellulose. In: Woodings C (ed) Regenerated cellulose fibres. Woodhead Publishing, Cambridge, pp 156-173

Hummel M, Michud A, Tanttu M et al (2015) Ionic liquids for the production of man-made cellulosic fibers: Opportunities and challenges. Adv Polym Sci 271:133-168. https:// doi.org/10.1007/12_2015_307

Jiang G, Huang W, Li L et al (2012) Structure and properties of regenerated cellulose fibers from different technology processes. Carbohydr Polym 87:2012-2018. https://doi. org/10.1016/j.carbpol.2011.10.022 
Lewis D, Zhao X, Tapley KN (2002) A new agent for cotton durable press finishing under alkaline conditions. AATCC Rev 2:38-41

Li F, Wang H, He J (2019) Synthesis and application of an alkaline crosslinking agent containing acrylamide as the durable press finishing agent on cotton fabric. Text Res $\mathbf{J}$ 89:2084-2097. https://doi.org/10.1177/004051751878 6271

Lim KY, Yun JS, Kim BC (2003) Reduction of fibrillation of Lyocell fiber with cellulose-g-poly(vinyl alcohol) copolymer. Polym J 35:691-696. https://doi.org/10.1295/polymj. 35.691

Liu J, Wang B, Xu X et al (2016) Green finishing of cotton fabrics using a xylitol-extended citric acid cross-linking system on a pilot scale. ACS Sustain Chem Eng 4:1131-1138. https://doi.org/10.1021/acssuschemeng. $5 \mathrm{~b} 01213$

Ma Y (2018) Fibre spinning from various low refined, recycledlignocelluloses using ionic liquid. Aalto Univeristy, Aalto

Manian AP, Aldred AK, Lenninger M, Bechtold T (2017) Alkali pretreatments and crosslinking of lyocell fabrics. Cellulose 24:3991-4002. https://doi.org/10.1007/s10570-017-13849

Maurer RJ, Sax AF, Ribitsch V (2013) Molecular simulation of surface reorganization and wetting in crystalline cellulose I and II. Cellulose 20:25-42. https://doi.org/10.1007/ s10570-012-9835-9

Michud A, King A, Parviainen A et al (2014) Process for the production of shaped cellulose articles. WO 2014/162062 A1

Mortimer S, Peguy A (1996a) The formation of structure in the spinning and coagulation of lyocell fibres. Cellul Chem Technol 30:117-132

Mortimer SA, Péguy AA (1996b) Methods for reducing the tendency of Lyocell fibers to fibrillate. J Appl Polym Sci 60:305-316. https://doi.org/10.1002/(sici)10974628(19960418)60:3<305::aid-app3>3.3.co;2-9

Patil NV, Netravali AN (2019) Cyclodextrin-based "green" wrinkle-free finishing of cotton fabrics. Ind Eng Chem Res 58:20496-20504. https://doi.org/10.1021/acs.iecr.9b04 092

Potter CD (1994) Fibre treatment. WO 94/09191

Potter CD, Dobson P (1995) Fibre treatment. WO 95/28516

Potter CD, Taylor JM (1998) Lyocell fabric treatment to reduce fibrillation tendency. EP0758415 A1

Röder T, Moosbauer J, Wöss K et al (2013) Man-made cellulose fibres-a comparison based on morphology and mechanical properties. Lenzinger B 91:7-12

Rohrer C, Retzl P, Firgo H (2001) Profile of a fibrillation-free fibre from Lenzing. Lenzinger Ber 80:75-81

Rojas J, Azevedo E (2011) Functionalization and crosslinking of microcrystalline cellulose in aqueous media: a safe and economic approach. Int J Pharm Sci Rev Res 8:28-36

Schurz J, Lenz J (1994) Investigations on the structure of regenerated cellulose fibers; Herrn Professor JaneschitzKriegl zum 70. Geburtstag mit den besten Wünschen gewidmet. Macromol Symp 83:273-289. https://doi.org/ 10.1002/masy.19940830123
Schuster KC, Rohrer C, Eichinger D et al (2004) Environmentally friendly Lyocell fibers. In: Wallenberger FT, Weston $\mathrm{NE}$ et al (eds) Natural fibers, plastics and composites. Springer, Boston, pp 123-146

Sixta H, Michud A, Hauru L et al (2015) Ioncell-F: a highstrength regenerated cellulose fibre. Nord Pulp Pap Res J 30:043-057. https://doi.org/10.3183/NPPRJ-2015-30-01p043-057

Stoyanoc A, Naieni S, Stephens S (2005) Glyoxal crosslinkinged cellulosic fibers having improved brightness and color. US 2005/0016698 A1

Talukder E, Manikandan N (2016) Optimum concentration of cross-linking agent for crease-resistant finishing using DMDHEU on knit and woven fabric in the field of ease care durable press. Int J Text Sci 5:74-81. https://doi.org/10. 5923/j.textile.20160504.02

Tomljenović A, Čunko R (2004) Reducing fibrillation tendency of man-made cellulose fibres employing ultrasound treatment. J Text Inst 95:327-339. https://doi.org/10.1533/joti. 2003.0059

Udoetok IA, Wilson LD, Headley JV (2018) "Pillaring Effects “ in cross-linked cellulose biopolymers: a study of structure and properties. Int J Polym Sci. https://doi.org/10.1155/ 2018/6358254

Udomkichdecha W, Chiarakorn S, Potiyaraj P (2002) Relationships between fibrillation behavior of Lyocell fibers and their physical properties. Text Res J 72:939-943. https://doi.org/10.1177/004051750207201101

Welch CM (1983) Glyoxal as a formaldehyde-free durable press reagent for mild curing applications'. Text Res J 53:181-186. https://doi.org/10.1177/004051758305300 307

Yang CQ, Wang X, Kang I-S (1997) Ester crosslinking of cotton fabric by polymeric carboxylic acids and citric acid. Text Res J 67:334-342. https://doi.org/10.1177/004051759706 700505

Yang CQ, Chen D, Guan J, He Q (2010) Cross-linking cotton cellulose by the combination of maleic acid and sodium hypophosphite. 1. Fabric wrinkle resistance. Ind Eng Chem Res 49:8325-8332. https://doi.org/10.1021/ie1007294

Yao W, Wang B, Ye T, Yang Y (2013) Durable press finishing of cotton fabrics with citric acid: Enhancement of whiteness and wrinkle recovery by polyol extenders. Ind Eng Chem Res 52:16118-16127. https://doi.org/10.1021/ ie $402747 x$

Ye T, Wang B, Liu J et al (2015) Quantitative analysis of citric acid/sodium hypophosphite modified cotton by HPLC and conductometric titration. Carbohydr Polym 121:92-98. https://doi.org/10.1016/j.carbpol.2014.12.028

Yu YH, Lee ES, Bang ES (2008) A new catalyst for glyoxal durable press finish of cotton fabrics. Fibers Polym 9:715-719. https://doi.org/10.1007/s12221-008-0112-3

Zhang W, Okubayashi S, Bechtold T (2005a) Fibrillation tendency of cellulosic fibers. Part 1: effects of swelling. Cellulose 12:267-273. https://doi.org/10.1007/s10570-0042786-Z

Zhang W, Okubayashi S, Bechtold T (2005b) Fibrillation tendency of cellulosic fibers. Part 2: effects of temperature. 
Cellulose 12:275-279. https://doi.org/10.1007/s10570004-2787-y

Zhu P, Liu J (2018) Preparation and properties of cross-linked regenerated cellulose fibers. J Text Sci Eng 8:1-5. https:// doi.org/10.4172/2165-8064.1000354

Zugenmaier P (2008) Crystalline cellulose and derivatives. Springer, Berlin
Publisher's Note Springer Nature remains neutral with regard to jurisdictional claims in published maps and institutional affiliations. 